\title{
Chemical Tumor Ablation with Use of a Novel Multiple-tine Infusion System in a Canine Sarcoma Model
}

\author{
Andrew Hines-Peralta, MD, Zheng-jun Liu, MD, Clare Horkan, MD, Stephanie Solazzo, BA, and \\ S. Nahum Goldberg, MD
}

PURPOSE: To determine whether larger confluent zones of ablation can be achieved in chemical ablation with use of a multiple-tine infusion device compared with standard needle infusion in a solid tumor model.

MATERIALS AND METHODS: Multiple canine venereal sarcomas $(N=42)$ were implanted in nine mildly immunosuppressed dogs (treated with $10 \mathrm{mg} / \mathrm{kg}$ cyclosporin A twice daily). Tumors incubated for 8-12 weeks grew to a diameter of $5.4 \mathrm{~cm} \pm 1.0$. With ultrasound guidance, $8-56 \mathrm{~mL}$ of $100 \%$ ethanol or $15 \%$ acetic acid (diluted in saturated saline solution) were injected in aliquots $(2-8 \mathrm{~mL})$ at multiple distances (radius of $0-2 \mathrm{~cm})$ from the needle axis with use of a multiple-tine infusion device. Presence of fluid reflux at the needle puncture site and resultant coagulation diameters were measured within 1 hour and compared with the results of infusion with a standard 18-gauge needle.

RESULTS: Multiple-tine infusion enabled greater fluid infusion ( $15 \mathrm{~mL} \pm 3$ to $53 \mathrm{~mL} \pm 3$ depending on protocol) than standard needle injection $(8 \mathrm{~mL} \pm 1)$ before reflux was observed at the puncture site $(P<.01)$. Additionally, progressive gains in contiguous tumor coagulation were achieved because acetic acid was infused as far as $2 \mathrm{~cm}$ from the needle axis with the multiple-tine device $\left(P<.01 ; R^{2}=0.59 ; y=0.5 x+2.9\right)$. Optimal coagulation was achieved with the infusion of 4-mL aliquots at $0.5 \mathrm{~cm}$ and $1.0 \mathrm{~cm}$ from the needle, followed by three $4-\mathrm{mL}$ or $8-\mathrm{mL}$ aliquots $\left(40^{\circ}\right.$ rotation between infusions) at $1.5 \mathrm{~cm}$ and $2.0 \mathrm{~cm}$ from the needle $(32 \mathrm{~mL} \pm 0$ and $53 \mathrm{~mL} \pm 3$ total, respectively). This yielded confluent short-axis coagulation diameters of $4.9 \mathrm{~cm} \pm 1.0$ and $5.4 \mathrm{~cm} \pm 1.0$, respectively, which were significantly greater than the measurement of $3.1 \mathrm{~cm} \pm 0.4$ achieved with standard needle infusion $(P<.01)$. Smaller and noncontiguous foci of coagulation foci $(1.7 \mathrm{~cm} \pm 0.5)$ were seen with the use of ethanol for standard needle and multiple-tine infusions.

CONCLUSIONS: Chemical ablation with 15\% acetic acid with use of a multiple-tine infusion device resulted in larger diameters of contiguous tumor coagulation and enabled greater volumes of infusion than standard needle infusion or ethanol ablation. This suggests that chemical ablation with acetic acid infused with use of a multiple-tine device may overcome some of the difficulties seen with the use of conventional needle chemical ablation injection alone, such as irregular ablation and fluid reflux up the needle tract.

J Vasc Interv Radiol 2006; 17:351-358

Abbreviation: $\quad R F=$ radiofrequency

CHEMICAL ablation with percutaneous ethanol instillation for small $(<3$ $\mathrm{cm}$ ) primary hepatocellular carcinomas has been shown to be as effective as surgical resection (1-5). However, many focal hepatomas are too large at diagnosis to be effectively treated with chemical ablation alone $(2,6,7)$. Disappointing results have also been reported for metastatic disease to the

From the Department of Radiology, Beth Israel Deaconess Medical Center, Harvard Medical School, 330 Brookline Avenue, Boston, Massachusetts 02215. Received June 16, 2005; accepted November 2. Address correspondence to S.N.G.; E-mail: sgoldber@caregroup.harvard.edu

Research supported in part by RexMedical, LP, Con- shohocken, PA. None of the authors have identified a conflict of interest.

(C) SIR, 2006

DOI: 10.1097/01.RVI.0000196355.31419.1E liver (8-11). This limitation has fueled the development of thermal tumor ablation methods, including radiofrequency (RF) (11-13), microwave (14), and cryoablation (15) devices.

The poor clinical performance of chemical ablation for larger tumors has been primarily attributed to unpredictable and irregular zones of coagulation achieved with large-volume chemical instillation, which in turn is caused by nonspherical, nonsymmetric diffusion of the agent via paths of least resistance. This leads to higher 
rates of local tumor progression compared with RF ablation despite an increase in the number of treatment sessions $(16,17)$. In addition, for dense solid tumors, the path of least resistance for chemical reflux may occur along the needle shaft (ie, the path of least resistance) into the peritoneum, leading to pain and other side effects (18). Irregular and unpredictable penetration with ethanol has also stimulated research into other chemical agents such as acetic acid, which has shown superior tissue penetration in laboratory studies $(19,20)$ and clinical studies (21).

One solution that has been proposed for RF ablation to overcome inadequate tissue coagulation is the development of a multiple-tine RF applicator (22). Several such devices are now commercially available. In this study, we apply this concept to chemical ablation as a possible means to improve chemical ablation diffusion by performing chemical ablation with use of a multiple-tine instillation device. In these studies, we first optimized a multiple-tine chemical ablation device for use in a solid canine sarcoma tumor model for acetic acid and ethanol. We then compared our results to those of standard needle infusion by assessing tumor coagulation, total infusible volume, and chemical reflux.

\section{MATERIALS AND METHODS}

\section{Overall Experimental Design}

Percutaneous chemical ablation was performed in large $(>5 \mathrm{~cm})$ canine venereal sarcoma tumors $(N=42$ in nine dogs) with a standard 18gauge needle $(n=6)$ or a multiple-tine device $(n=36)$. Ablation was performed with $100 \%$ ethanol $(n=9)$ or $15 \%$ acetic acid diluted in a saturated saline solution $(n=33)$, a mixture that has been shown to have antitumor effects in this animal model (19). With the multiple-tine device, chemical instillation was performed by injecting 2-8-mL aliquots at multiple distances $(0-2 \mathrm{~cm})$ perpendicular to the needle axis. Resultant coagulation diameters, quality of ablation (ie, confluence and shape), total infusible volume, and fluid reflux were compared versus the results of standard 18-gauge needle infusion.

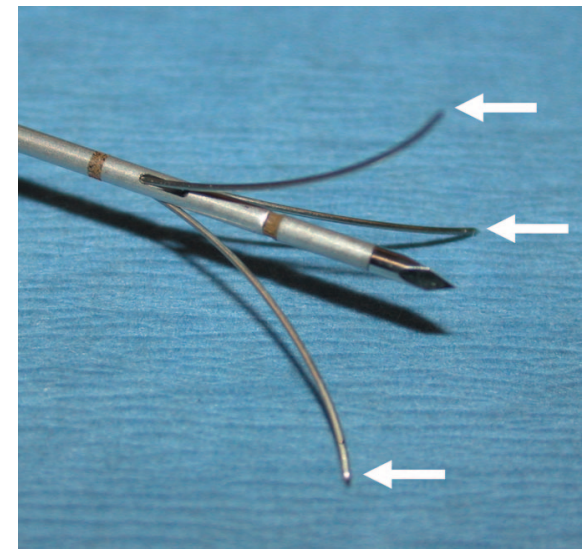

Figure 1. Multiple-tine chemical ablation infusion device. The three tines extending from the trocar shaft (arrows) are deployed to a diameter of $2 \mathrm{~cm}$ around the needle axis. This lateral extension enables delivery of sclerosant deeper into the tissues.

\section{Device Design}

Chemical infusion was performed with use of a multiple-tine infusion device consisting of three deployable tines embedded within an 18-gauge shaft (QuadraFuse; RexMedical, Conshohocken, PA; Fig 1). The tines are separated by $120^{\circ}$ and deploy in an umbrella fashion. Infusion of a chemical agent occurs simultaneously through the end of each tine at a radial distance $0.5-2 \mathrm{~cm}$ perpendicular to the needle shaft depending on how far the tines are deployed. The deployment settings described here refer to the diameter distance between tines (deployed 1-4 cm). Given the design, the tines also travel approximately $1 \mathrm{~cm}$ distally along the needle axis direction (ie, $\mathrm{z}$ axis) as they are deployed from the $1-\mathrm{cm}$ setting to the $4-\mathrm{cm}$ setting. The ablative agent is delivered manually via a syringe attached to the device.

\section{Tumor Preparation and Animal Care}

Use of animals was conducted through the approval of the institutional animal care and use committee. Multiple canine venereal sarcomas were implanted subcutaneously on the back and torso in a canine model. Canine venereal sarcoma cell line has well-characterized growth and has been used for chemical ablation and RF ablation studies $(20,23)$. Female mongrel dogs $(N=9$; Harlan Farms,
North Rose, NY) were mildly immunosuppressed $(10-20 \mathrm{mg} / \mathrm{kg}$ cyclosporin A twice daily; Neoral; Novartis, East Hanover, NJ) 5 days before tumor transplantation until the end of the experiment. Under sterile technique, tumor from a live carrier was harvested and homogenized with a tissue grinder (Model 23; Kontes Glass, Vineland, NJ) and suspended in Dulbecco Modified Eagle Medium (INC Biomedicals, Aurora, IL) to a concentration of approximately $1 \times 10^{8}$ cells / $\mathrm{mL}$. Animals were anesthetized with Telazol (Lederle, Carolina, PR), and tumor injection sites were shaved and disinfected with Betadine (Purdue Frederick, Norwalk, CT) and 100\% ethanol (Fisher Scientific, Fairlawn, NJ). The tumor suspension was injected under direct visualization via an 18-gauge needle into 12 sites on the back and torso. The tumors were allowed to grow to a mean diameter of $5.4 \mathrm{~cm} \pm 1.0$, approximately $10-12$ weeks after inoculation. Animals were monitored daily and tumor sizes were measured weekly. Adequate tumor yield per animal (tumor diameter $\geq 4$ cm) was approximately $33 \%-67 \%$ (four to eight tumors per 12 injected), given variation in tumor growth and shape.

\section{Tumor Size}

The tumors reached a mean diameter of $5.4 \mathrm{~cm} \pm 1.0$ (short-axis diameter range, 3.9-6.2 cm; long-axis diameter range, $4.8-7.5 \mathrm{~cm}$ ). All tumors were solid with a thin surrounding membrane. Histologically, the tumors were composed of a dense cellular stroma. These tumors are hypovascular tumors with more vascularity outside the surrounding membrane. Tumor appearance was similar to that seen in previous studies (20).

\section{Chemical Ablation}

Two chemical agents, acetic acid and ethanol, were used to perform chemical ablation. One hundred percent ethanol and $50 \%$ acetic acid were acquired from the manufacturer (Fisher Scientific). Ethanol was used as supplied, but the $15 \%$ acetic acid solution was created through the dilution of $50 \%$ stock solution in saturated $36 \%$ saline solution until a $15 \%$ acetic acid concentration was achieved. This dilu- 


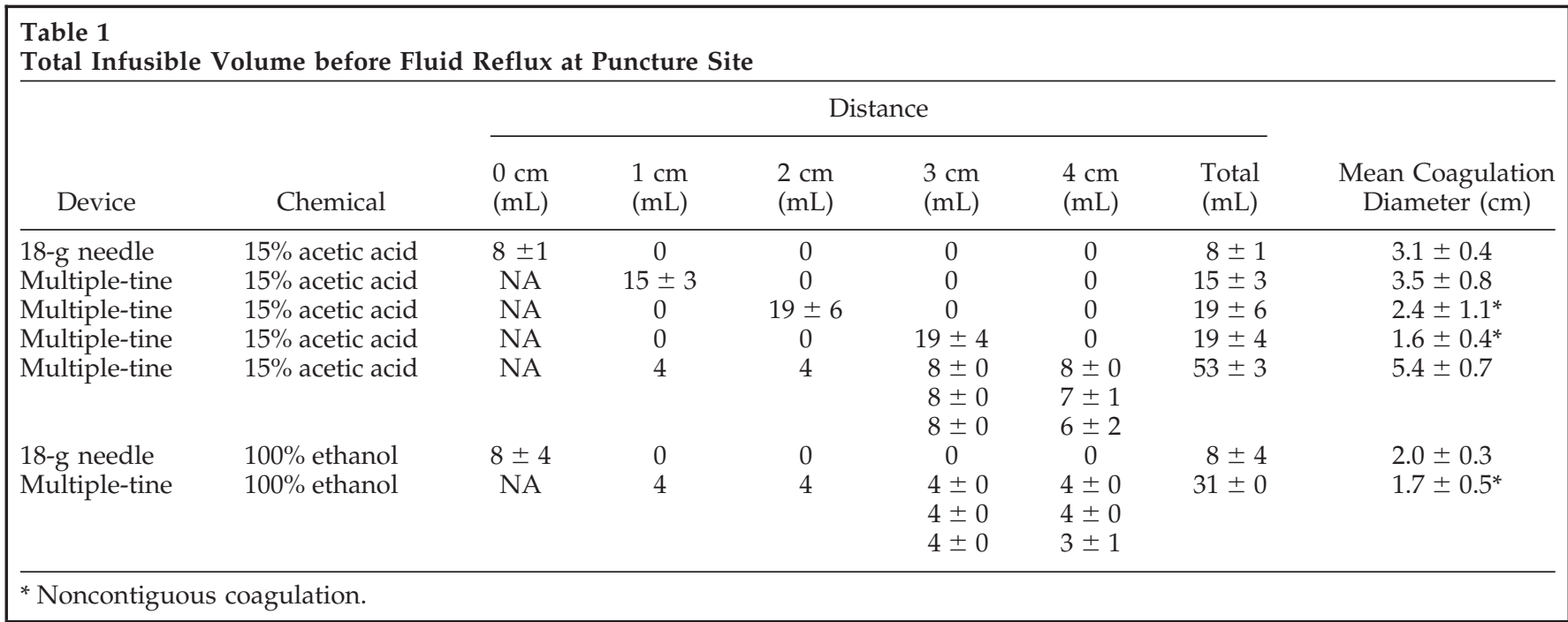

tion mixture was chosen based on optimal results achieved previously in this tumor model (20).

Percutaneous multiple-tine infusion was performed under ultrasound (US) guidance. Tine deployment and proper tine spacing was confirmed by US. Infusion of the entire volume was performed according to a stepwise injection strategy comprising various combination deployments including diameters of $1 \mathrm{~cm}, 2 \mathrm{~cm}, 3 \mathrm{~cm}$, and 4 $\mathrm{cm}$ perpendicular to the needle axis. For 1-cm and 2-cm tine deployment, a single aliquot injection was used. However, for infusion at distances of 3 $\mathrm{cm}$ and $4 \mathrm{~cm}$, three aliquots were infused, allowing for $40^{\circ}$ tine rotation among aliquots to enable superior distribution (based on unpublished preliminary data). The total volume of chemical agent infused per tumor ranged from $8 \mathrm{~mL}$ to $56 \mathrm{~mL}$.

Percutaneous standard needle infusion was performed with use of a standard 18-gauge, 3-cm needle (Fisher Scientific) that was introduced under US guidance into the center of the tumor. For all needle types, each bolus of $100 \%$ ethanol or $15 \%$ acetic acid was slowly infused over a period of $30 \mathrm{sec}-$ onds with use of a 10-mL syringe. Infusion was discontinued when reflux of fluid was observed at the surface of the tumor at the needle puncture site.

\section{Pathologic Studies of Necrosis}

Each subject animal was euthanized immediately after ablation and tumors were removed and sectioned within 1 hour after the animal's death. Tumors were sectioned perpendicular to the device axis in 1-cm-thick slabs with use of a sharpened tissue-sectioning knife. Immediately after sectioning, mitochondrial enzyme activity was used as an indicator of tumor viability, and was assessed by incubating tissue sections for 30 minutes in $2 \%$ 2,3,5-triphenyl tetrazolium chloride (Fisher Scientific) at $20^{\circ} \mathrm{C}-25^{\circ} \mathrm{C}$. The absence of mitochondrial enzyme activity has been shown to accurately reveal irreversible cellular injury induced by many forms of percutaneous tumor ablation (24). After the 30minute incubation, the extent of visible gross coagulation in the short axis diameter (ie, perpendicular to the needle axis) was then measured with calipers in cross-sectioned $\mathrm{x}$ and $\mathrm{y}$ axes. Tissue that did not visibly stain for mitochondrial enzyme was defined as coagulated as per previous studies (24).

\section{Statistical Analysis}

For all experiments, each parameter was fulfilled three separate times in separate tumors, and all findings are reported as means \pm SD. Primary outcome measures included total volume infusible before visualization of fluid reflux at the needle puncture site. Another primary outcome measure used for statistical analyses included coagulation diameter perpendicular to the applicator shaft (ie, short axis) (25). In tumors with asymmetric shapes, the $x$ axis was defined as the longest crosssectional diameter from the analyzed slabs. The y axis diameter was defined as the perpendicular coagulation diameter of the same slab. Student $t$ tests (significance threshold of $P=.05$; twotailed test; Excel 2002; Microsoft, Redmond, WA) or analysis of variance (version 6.1; Origin, Northampton, MA) was used to assess significant difference for comparison groups as appropriate. Multivariate regressions with use of linear and higher-order regression models were performed with use of Origin 6.1 software with the strength of the best-fit regression curves reported as $\mathrm{R}^{2}$ computations.

\section{RESULTS}

\section{Fluid Reflux}

Multiple-tine infusion at $1 \mathrm{~cm}$ deployment enabled significantly more acetic acid to be infused $(15 \mathrm{~mL} \pm 3)$ than standard needle infusion $(8 \mathrm{~mL} \pm$ $1 ; P<.01)$ before reflux of fluid was observed at the tumor surface (Table 1). However, single infusions at greater deployment distances did not enable significantly more fluid to be infused without reflux (Table 1). The greatest amount of infusible fluid was obtained with use of the stepped aliquot infusion protocol (as described later) in which greater total infusion volumes could be infused as further deployments were performed. The total infusible volume with a stepped 


\begin{tabular}{|c|c|c|c|c|c|c|}
\hline \multicolumn{7}{|c|}{$\begin{array}{l}\text { Table } 2 \\
\text { Optimization of Multiple-tine Infusion for 15\% Acetic Acid }\end{array}$} \\
\hline \multirow[b]{2}{*}{$\begin{array}{l}\text { Tumors } \\
\text { Treated }\end{array}$} & \multicolumn{5}{|c|}{ Distance } & \multirow[b]{2}{*}{$\begin{array}{c}\text { Mean Coagulation } \\
(\mathrm{cm})\end{array}$} \\
\hline & $\begin{array}{l}1 \mathrm{~cm} \\
(\mathrm{~mL})\end{array}$ & $\begin{array}{l}2 \mathrm{~cm} \\
(\mathrm{~mL})\end{array}$ & $\begin{array}{l}3 \mathrm{~cm} \\
(\mathrm{~mL})\end{array}$ & $\begin{array}{l}4 \mathrm{~cm} \\
(\mathrm{~mL})\end{array}$ & $\begin{array}{l}\text { Total } \\
(\mathrm{mL})\end{array}$ & \\
\hline 3 & $4 \pm 0$ & $4 \pm 0$ & 0 & 0 & $8 \pm 0$ & $3.2 \pm 0.9$ \\
\hline 3 & 0 & $12 \pm 0$ & $4 \pm 0$ & 0 & $16 \pm 0$ & $3.2 \pm 1.0^{*}$ \\
\hline 3 & $4 \pm 0$ & $4 \pm 0$ & $4 \pm 0$ & $4 \pm 0$ & $16 \pm 0$ & $3.3 \pm 1.0^{*}$ \\
\hline 3 & $4 \pm 0$ & $4 \pm 0$ & $8 \pm 0$ & $8 \pm 0$ & $24 \pm 0$ & $3.8 \pm 1.2^{*}$ \\
\hline 3 & $4 \pm 0$ & $4 \pm 0$ & $\begin{array}{l}2 \pm 0 \\
2 \pm 0 \\
2 \pm 0\end{array}$ & $\begin{array}{l}2 \pm 0 \\
2 \pm 0 \\
2 \pm 0\end{array}$ & $20 \pm 0$ & $4.0 \pm 0.3^{*}$ \\
\hline 3 & $4 \pm 0$ & $4 \pm 0$ & $\begin{array}{l}4 \pm 0 \\
4 \pm 0 \\
4 \pm 0\end{array}$ & $\begin{array}{l}4 \pm 0 \\
4 \pm 0 \\
4 \pm 0\end{array}$ & $32 \pm 0$ & $4.9 \pm 1.1$ \\
\hline 3 & $4 \pm 0$ & $4 \pm 0$ & $\begin{array}{l}8 \pm 0 \\
8 \pm 0 \\
8 \pm 0\end{array}$ & $\begin{array}{l}8 \pm 0 \\
7 \pm 1 \\
6 \pm 2\end{array}$ & $53 \pm 3$ & $5.4 \pm 0.7$ \\
\hline
\end{tabular}

* Noncontiguous ablation.

Note.-Triplicate numbers at $3 \mathrm{~cm}$ and $4 \mathrm{~cm}$ deployment denote that the device was rotated $40^{\circ}$ among three smaller aliquot infusions at that diameter.

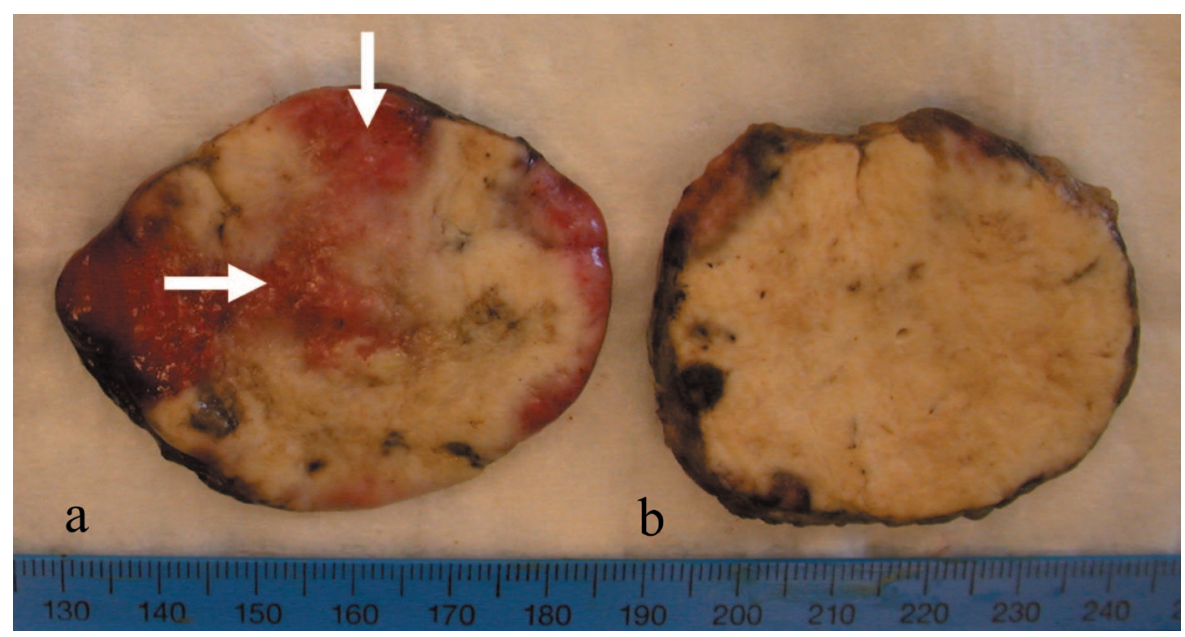

Figure 2. Achievement of contiguous coagulation with $15 \%$ acetic acid. (a) Single infusion at $3 \mathrm{~cm}$ of $19 \pm 4 \mathrm{~mL}$ results in nonconfluent coagulation with viable patches of tissue (red tissue; arrows) observed throughout the ablation. Conversely, stepped infusions at $1 \mathrm{~cm}(4 \mathrm{~mL}), 2 \mathrm{~cm}(4 \mathrm{~mL}), 3 \mathrm{~cm}(12 \mathrm{~mL}), 4 \mathrm{~cm}(12 \mathrm{~mL} ; 32 \mathrm{~mL}$ total) produce large zones of confluent coagulation in this hard tumor model (b).

aliquot approach to a maximum deployment of $4 \mathrm{~cm}$ was $53 \mathrm{~mL} \pm 3$.

For ethanol, standard needle infusion of $100 \%$ ethanol enabled infusion of $8 \mathrm{~mL} \pm 4$ before reflux was noted at the puncture site. More total infusible volume was achievable with use of the multiple-tine device $(P<.05)$ in a stepped aliquot approach in which 31 $\mathrm{mL} \pm 1$ represented the limit of infusible volume without reflux. The greatest infusion possible with $100 \%$ ethanol $(31 \mathrm{~mL} \pm 1)$ was notably less than

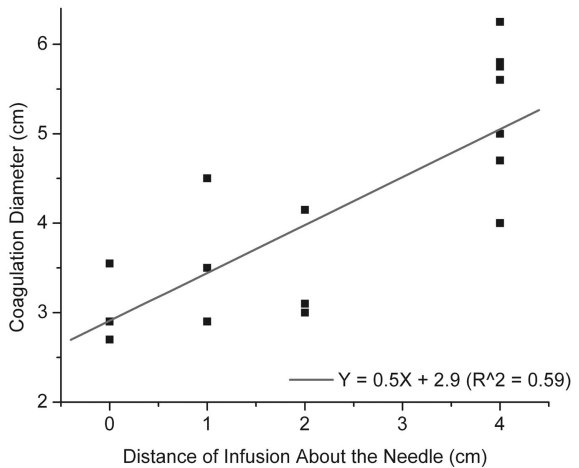

Figure 3. With stepped aliquot infusion of $15 \%$ acetic acid, the needle and resultant coagulation diameter with use of the multiple-tine device increases linearly with the increase in chemical volume. Use of greater deployment distances enabled greater total infusion volume, resulting in larger zones of coagulation.

(Fig 2). To achieve contiguous ablation for $2-\mathrm{cm}, 3-\mathrm{cm}$, and $4-\mathrm{cm}$ tine deployment, (ie, tines extending $1.0-2.0 \mathrm{~cm}$ from the central cannula), additional infusions were necessary at $1 \mathrm{~cm}$ and 2 $\mathrm{cm}$ from the needle tip; otherwise, central residual viable tumor was seen at the center of the tumor (Fig 2). At the $3-\mathrm{cm}$ and $4-\mathrm{cm}$ settings, three aliquots (with tine rotation of $40^{\circ}$ among aliquots) were needed to achieve round confluent coagulation, as single aliquot infusions at the $2-\mathrm{cm}$ and $3-\mathrm{cm}$ settings yielded irregular borders. Optimal infusions (ie, continuous coagulation without reflux) in this hard tumor model for spherical confluent chemical ablation were $4 \mathrm{~mL}$ for $1-\mathrm{cm}$ and 2-cm deployments and three 4-mL $40^{\circ}$ rotated aliquots at $3 \mathrm{~cm}$ and $4 \mathrm{~cm}$ (Table 2).

\section{Comparison of Extent of Coagulation}

Significant gains in coagulation were achieved as more fluid was infused with use of our optimized multiple-aliquot algorithm at further distances from the needle axis $(P<.05$; linear $\mathrm{R}^{2}=0.59$; Figs 3-4). With an optimal multiple-tine infusion protocol of $32 \mathrm{~mL}$ and $15 \%$ acetic acid infusion $(1 \mathrm{~cm}, 4 \mathrm{~mL} ; 2 \mathrm{~cm}, 4 \mathrm{~mL} ; 3 \mathrm{~cm}$, three $4-\mathrm{mL}$ aliquots at $40^{\circ}$ rotation; 4 $\mathrm{cm}$, three $4-\mathrm{mL}$ aliquots at $40^{\circ}$ rotation), the mean coagulation diameter measured $4.9 \mathrm{~cm} \pm 1.1$, which was significantly larger than the coagula- 


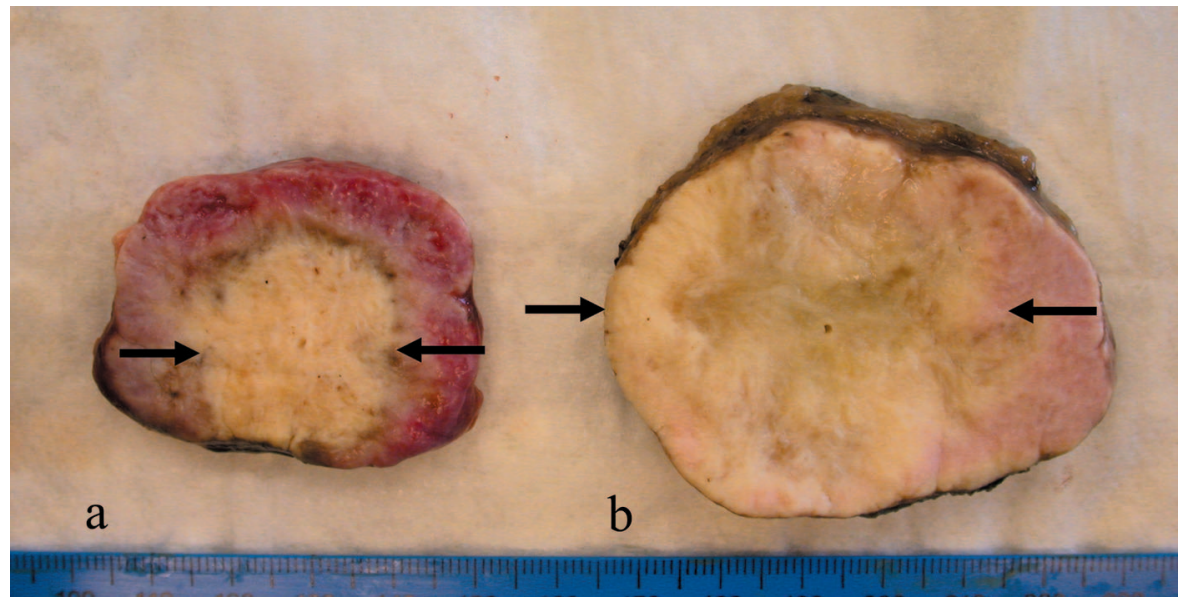

Figure 4. (a) Multiple-tine infusion with $15 \mathrm{~mL} \pm 3$ of $15 \%$ acetic acid at the 1 -cm setting. (b) Stepped multiple-tine infusion with the following protocol: $1 \mathrm{~cm}, 4 \mathrm{~mL} ; 2 \mathrm{~cm}, 4 \mathrm{~mL}$; $3 \mathrm{~cm}, 12 \mathrm{~mL} ; 4 \mathrm{~cm}, 12 \mathrm{~mL}$ (32 mL total). Infusion further away from the needle enables more total infusion and yields a significantly larger and confluent lesion.

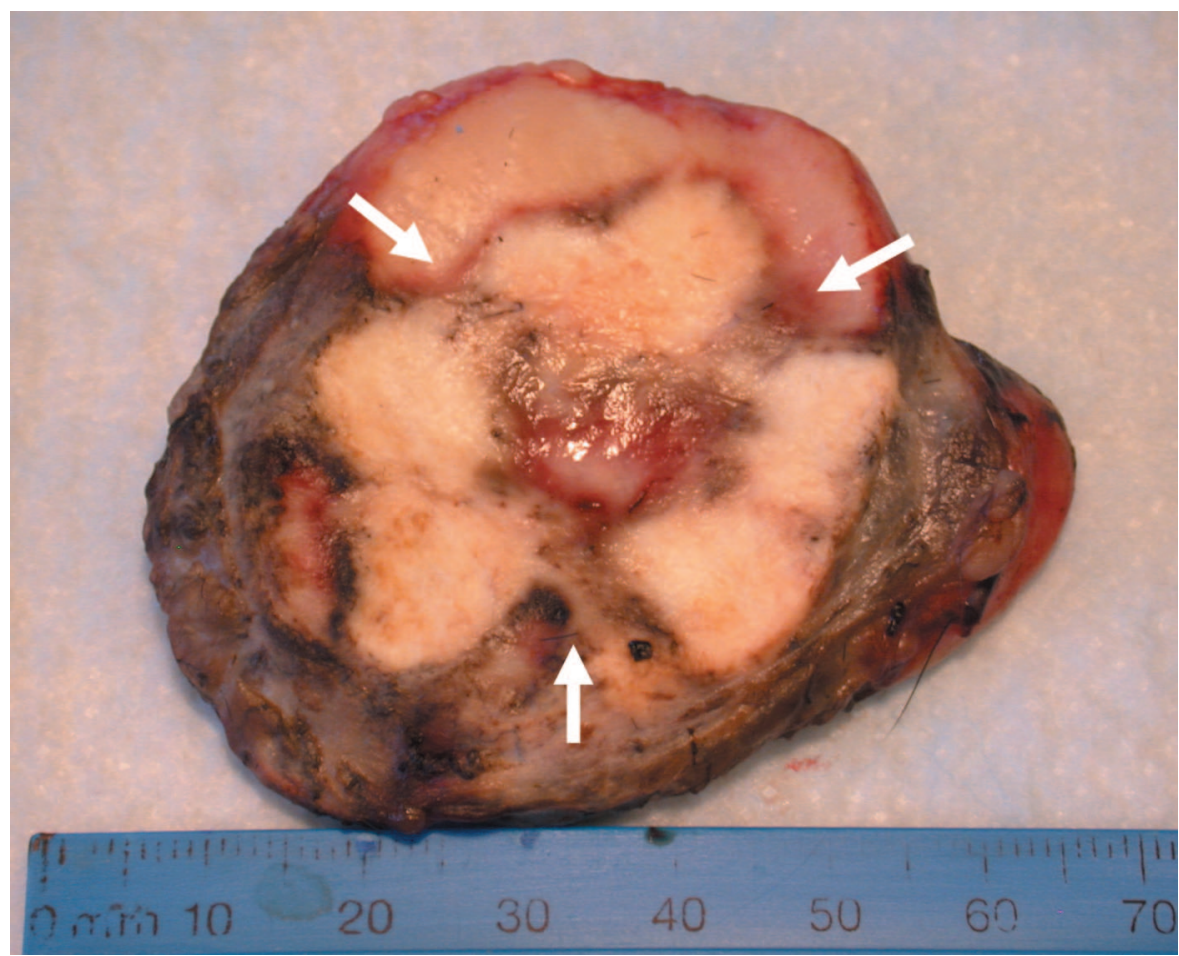

Figure 5. Ethanol ablation: in this hard tumor model, treatment with $100 \%$ ethanol with an optimal multiple-tine infusion protocol ( $32 \mathrm{~mL}$, stepped delivery) results in a cloverleaf pattern of foci of ablation (arrows).

tion diameter with maximum standard needle infusion of $8 \mathrm{~mL} \pm 1$, which yielded $3.1 \mathrm{~cm} \pm 0.4(P<.01$; Table 3).

Fifteen percent acetic acid achieved significantly larger and more confluent zones of ablation than $100 \%$ ethanol for standard needle infusion and optimal 32-mL multiple-tine infusion. three small $(1.7 \mathrm{~cm} \pm 0.5)$ separate zones of coagulation (Fig 5) in a cloverleaf pattern. These areas of chemical ablation corresponded to the paths of the three tines.

Multiple-tine infusion of $15 \%$ acetic acid also resulted in more spherical zones of ablation than standard needle injection alone. Injection via a standard single needle of $8 \mathrm{~mL} \pm 1$ (ie, maximum infusible volume without reflux) resulted in elliptical coagulation measuring $3.9 \mathrm{~cm} \pm 1.0$ (along the needle shaft) by $2.2 \mathrm{~cm} \pm 0.3$ in diameter. However, infusion of $8 \mathrm{~mL}$ with use of the multiple-tine device $(1 \mathrm{~cm}, 4$ $\mathrm{mL} ; 2 \mathrm{~cm}, 4 \mathrm{~mL}$ ) created a relatively spherical lesion measuring $3.2 \mathrm{~cm} \pm$ 0.9 by $3.0 \mathrm{~cm} \pm 0.9$.

\section{Adverse Effects}

Some local adverse effects were observed after infusion of $53 \mathrm{~mL} \pm 3$ acetic acid (ie, 8-mL aliquots at $3 \mathrm{~cm}$ and $4 \mathrm{~cm}$ ). Specifically, sclerosis of normal blood vessels at the base of tumor was observed in $67 \%$ of cases (two of three ablations), suggesting that systemic chemical reflux was occurring with use of the larger $8-\mathrm{mL}$ aliquots (Fig 6). No adverse effects associated with chemical uptake were observed under all other infusion protocols.

\section{DISCUSSION}

\section{Multiple-tine Infusion Results in Greater Total Infused Volume}

The data suggest that larger zones of ablation can be achieved with larger volumes of injected sclerosing compounds (26). However, for hard tumor models, the total amount of infusible volume is limited by chemical reflux outside the tumor, which usually occurs at the needle puncture site. In the clinical setting, this results in unwanted spillage of ablative agents into the peritoneal cavity or surrounding tissue, causing pain or other negative side effects (18). The data here suggest that multiple-tine infusion with use of a stepped aliquot protocol reliably enables significantly more infusible volume while minimizing the risk of chemical reflux. The result is significantly greater coagulation with more predictable and spherical contours. Standard needle infusion is likely lim- 


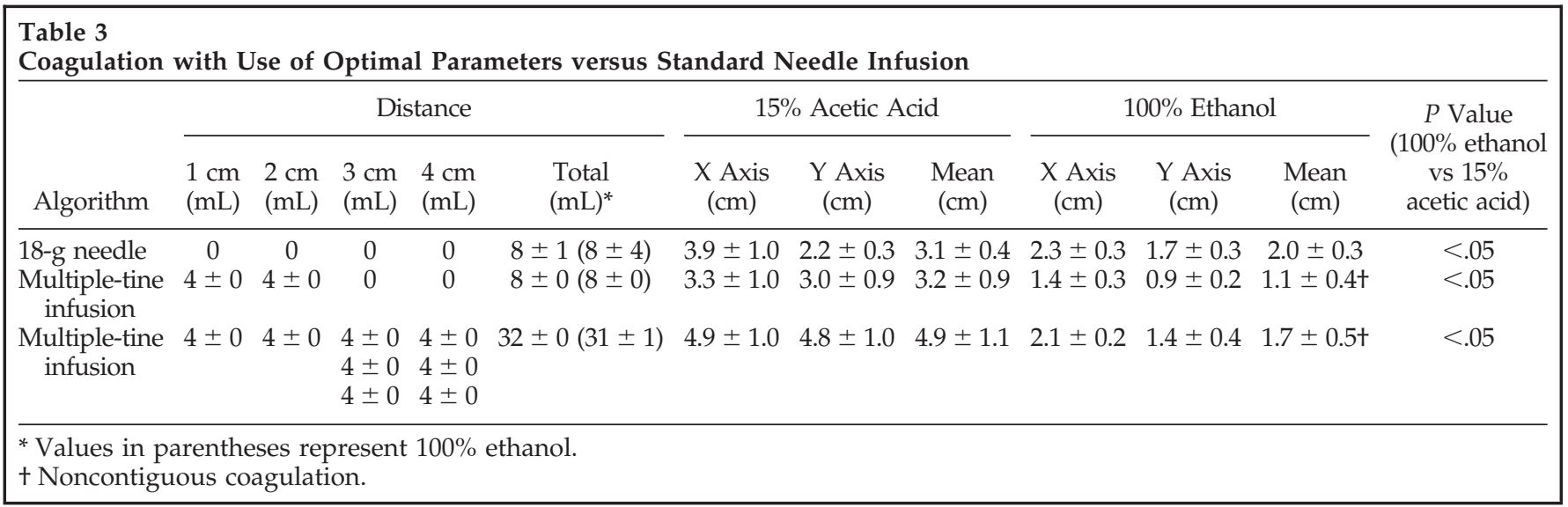

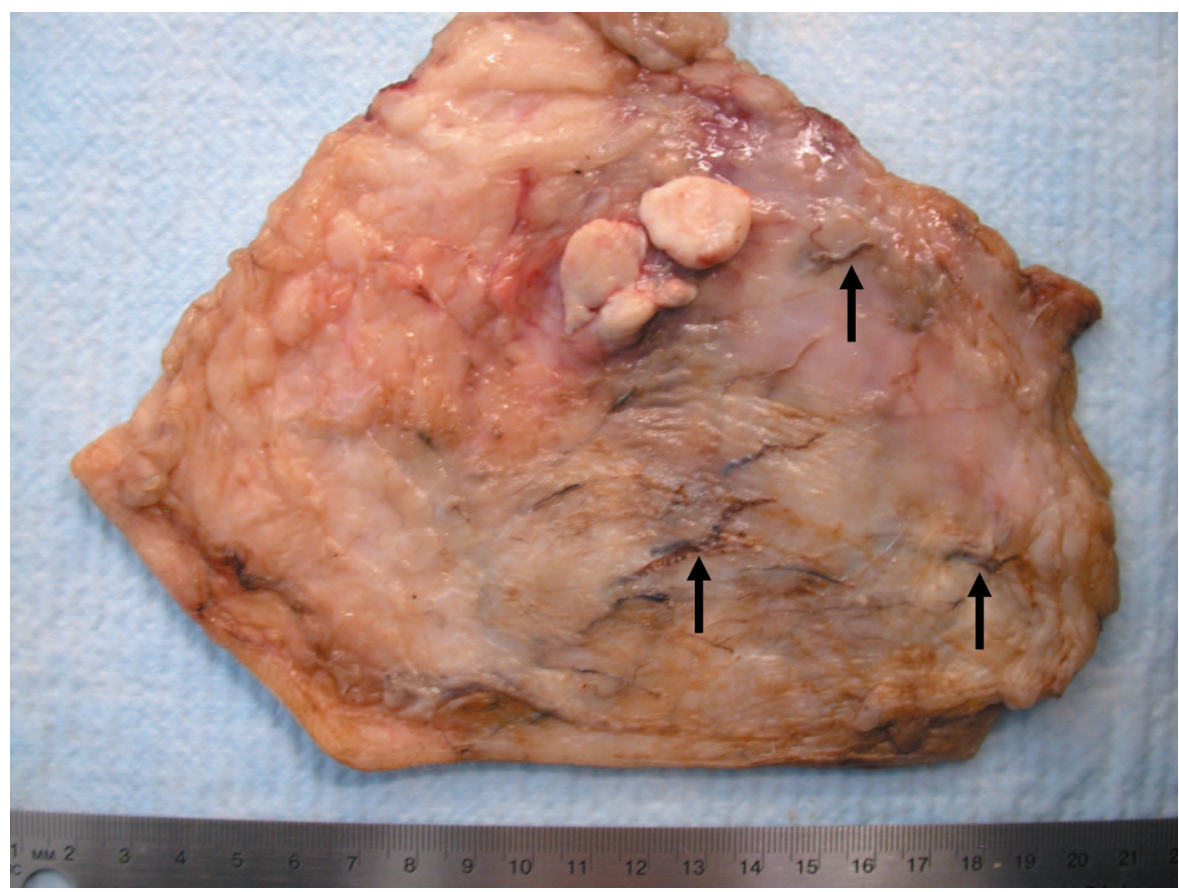

Figure 6. Skin specimens that overlay a tumor treated with multiple-tine infusion of 53 $\mathrm{mL} \pm 3$ of $15 \%$ acetic acid. Sclerosis of the vessels (arrows) in the skin surrounding the tumor occurs from uptake of acid in the skin, a negative side effect of treatment. This finding suggests that one must be judicious not to overinject chemical agents.

ited because fluid easily tracks back along the needle axis when high resistance is encountered in tumor parenchyma. This problem is potentially compounded because this fluid induces chemical sclerosis in tissues adjacent to the needle tract. However, in multiple-tine infusion, multiple diffusion paths are simultaneously created away from the needle axis, enabling greater penetration into tumor parenchyma. Stepwise extension of the tines further brings the chemical agent to new areas of the tumor that have not undergone sclerosis.

\section{Acetic Acid versus Ethanol Chemical Ablation}

Acetic acid diluted to $15 \%$ in saturated saline solution was far superior to $100 \%$ ethanol for standard needle infusion and multiple-tine infusion. This supports previous data indicating that even dilute acetic acid achieves superior coagulation to $100 \%$ ethanol
(19-21). The larger confluent zones of coagulation from $15 \%$ acetic acid likely result from superior penetration and diffusion into the solid tumor parenchyma. This contrasts with multiple-tine infusion of $100 \%$ ethanol, which yielded small zones of noncontiguous coagulation surrounding tine deployment sites. This suggests that, in some hard tumor types, ethanol is not sufficient to achieve adequate treatment even when aided by simultaneous multiple-tine infusion.

Although the greatest coagulation occurred with greatest infused volume (53 $\mathrm{mL} \pm 3)$, this was also associated with adverse events. As expected, this suggests that there is an upper boundary of safety even for this technique. The maximum safe infusible volume for chemical infusion may be higher in soft hepatocellular carcinoma, other tumor types, or larger tumors, but nevertheless, it may be prudent to gain initial clinical experience with the use of protocols like the one suggested herein. Similar recommendations are reported by other investigators for ethanol infusion (1-3). Regardless, it is most important to determine the minimal amount of any chemical necessary to achieve satisfactory results to minimize the possibility of these adverse risks.

\section{Limitations of the Study}

Given a limited number of available tumors to study, we assessed only two concentrations of ablation fluid, $100 \%$ ethanol and $15 \%$ acetic acid diluted in saturated saline solution, and a finite number of injection algorithms. Therefore, it is possible that 
further modification of the ethanol or acetic acid regimen may yield superior results. In addition to different infusion fluid, the tumor type itself is likely to influence the size and quality of ablation for multiple-tine infusion, given that standard needle infusion yields different results for hepatocellular carcinoma and colorectal carcinoma $(2,3,10)$. Results will need to be refined for each tumor and clinical scenario.

Size limitations of our model permitted study of tine extension to no greater than $4 \mathrm{~cm}$ because our tumor sizes were almost always limited to a short-axis diameter of $4-5 \mathrm{~cm}$ given animal care committee stipulations. Fortunately, these sizes are currently at the upper limits of tumors in which chemical ablation treatment can achieve favorable clinical results. In addition to tumor type, tumor size is likely to affect the total infusible volume and coagulation size. Therefore, further clinical study will be needed to determine the effect of tumor size and type as well as the optimal number and volume of injection aliquots for 5 -cm deployment. In these experiments, calculation of the $\mathrm{z}$ axis of coagulation (ie, along the needle axis) would also be advantageous to enable calculation of the volume of ablation in addition to the coagulation diameters.

In addition, we did not assess coagulation, allowing for repeat infusion after repositioning of a standard needle, which is commonly performed in clinical practice. In such an experiment, allowing for repositioning introduces multiple variables that may be difficult to assess given the limited availability for large animal studies. A future area of interest may be the comparison of single or multiple multipletine infusions versus multiple repositioned standard needle infusions in a clinical study. Under such circumstances, we hypothesize that repetitive needle repositioning may lead to more irregular results and necessitate treatment with more total infusion volume to achieve similar results. In addition to this, clinical outcome research and cost-benefit studies will likely be warranted, as the very low costs of standard needle infusion must be weighed against the higher cost of precision chemical ablation with various niche chemical ablation methods such as the multiple-tine infusion investigated here. Ultimately, comparison of outcomes, cost effectiveness, and physician and patient preferences will need to be compared for these chemical ablation devices against other ablation techniques such as RF and microwave ablation. This will likely reveal various strengths of individual modalities for the treatment of different tumor types.

\section{CONCLUSION}

Multiple-tine chemical infusion improved the size and quality of chemical ablation compared with standard needle infusion. In a large solid animal tumor, this suggests that chemical ablation with acetic acid infused with a multiple-tine device may yield a larger extent of coagulation than conventional needle injection alone in clinical practice, even for some hard solid tumors. Given the significant superiority of $15 \%$ acetic acid compared with $100 \%$ ethanol, it is probably worthwhile to continue to pursue infusion strategies with the use of acetic acid, most likely with the optimized injection algorithm reported herein as a starting point. More investigation is necessary to determine if the precise infusion achieved with multiple-tine devices enables more effective and efficient volume use than repositioning a standard single needle. However, use of the single needle stick offered by this multiple-tine device is likely to be more straightforward and potentially will reduce the procedure time, and theoretically can reduce the risks of some complications such as bleeding. As such, further research in the form of preliminary clinical trials with use of a stepped algorithm as suggested herein is likely warranted to determine the utility of multiple-tine chemical ablation in the clinical setting.

\section{References}

1. Livraghi T, Giorgio A, Marin G, et al. Hepatocellular carcinoma and cirrhosis in 746 patients: long-term results of percutaneous ethanol injection. Radiology 1995; 197:101-108.

2. Shiina S, TagawaK, Niwa $Y$, et al Percutaneous ethanol injection therapy for hepatocellular carcinoma: results in 146 patients. AJR Am J Roentgenol 1993; 160:1023-1028.

3. Lencioni R, Pinto F, Armillotta N, et al. Long-term results of percutaneous ethanol injection therapy for hepatocellu- lar carcinoma in cirrhosis: a European experience. Eur Radiol 1997; 7:514-519.

4. Livraghi T. Radiofrequency ablation, PEIT, and TACE for hepatocellular carcinoma. J Hepatobiliary Pancreat Surg 2003; 10:67-76.

5. Lencioni R, Cioni D, Crocetti L, et al. Percutaneous ablation of hepatocellular carcinoma: state-of-the-art. Liver Transpl. 2004; 10(2 suppl 1):S91-S97.

6. Livraghi T. Percutaneous ethanol injection in the treatment of hepatocellular carcinoma in cirrhosis. Hepatogastroenterology 2001; 48:20-24.

7. Lencioni R, Pinto F, Armillotta N, et al. Long-term results of percutaneous ethanol injection therapy for hepatocellular carcinoma in cirrhosis: a European experience. Eur Radiol 1997; 7:514-519.

8. McGahan JP, Dodd GD III. Radiofrequency ablation of liver tumors: current status. AJR Am J Roentgenol 2001; 176:3-16.

9. Gillams AR, Lees WR. Survival after percutaneous, image-guided, thermal ablation of hepatic metastases from colorectal cancer. Dis Colon Rectum 2000; 43:656-661.

10. Giovannini M, Seitz JF. Ultrasoundguided percutaneous alcohol injection of small liver metastases: results in 40 patients. Cancer 1994; 73:294-7.

11. Liu Z, Lobo SM, Humphries S, et al. Radiofrequency tumor ablation: insight into improved efficacy using computer modeling. AJR Am J Roentgenol 2005; 184:1347-1352.

12. Pereira PL, Trubenbach J, Schenk M, et al. Radiofrequency ablation: in vivo comparison of four commercially available devices in pig livers. Radiology. 2004; 232(2):482-490.

13. Rhim H, Goldberg SN, Dodd GD III, et al. Essential techniques for successful radio-frequency thermal ablation of malignant hepatic tumors. Radiographics 2001; 21(suppl):S17-S35.

14. Shock SA, Meredith K, Warner TF, et al. Microwave ablation with loop antenna: in vivo porcine liver model. Radiology. 2004; 231:143-149.

15. Lau WY, Leung TW, Yu SC, et al. Enhanced renal parenchymal cryoablation with novel 17-gauge cryoprobes. Urology 2004; 64:173-175.

16. Arata S, Tanaka $\mathrm{K}$, Okazaki $\mathrm{H}$, et al. Risk factors for recurrence of large HCC in patients treated by combined TAE and PEI. Hepatogastroenterology 2001; 48:480-485.

17. Ikeda M, Okada S, Ueno H, et al. Radiofrequency ablation and percutaneous ethanol injection in patients with small hepatocellular carcinoma: a comparative study. Jpn J Clin Oncol. 2001; 31:322-326. 
18. Vehmas T. Reflux of ethanol during experimental liver ethanol injections. Invest Radiol. 1992; 27:918-921.

19. Shah SS, Jacobs DL, Krasinkas AM, et al. Percutaneous ablation of VX2 carcinoma-induced liver tumors with use of ethanol versus acetic acid: pilot study in a rabbit model. J Vasc Interv Radiol 2004; 15:63-67.

20. Ahmed M, Weinstein J, Liu Z, et al. Image-guided percutaneous chemical and radiofrequency tumor ablation in an animal model. J Vasc Interv Radiol 2003; 14:1045-1052.

21. Huo TI, Huang $\mathrm{YH}, \mathrm{Wu} J \mathrm{C}$, et al. Comparison of percutaneous acetic acid injection and percutaneous ethanol injection for hepatocellular carcinoma in cirrhotic patients: a prospective study. Scand J Gastroenterol 2003; 38:770-778.

22. Rossi S, Buscarini E, Garbagnati F. Percutaneous treatment of small hepatic tumors by an expandable RF needle electrode. AJR Am J Roentgenol 1998; 170:1015-1022.

23. Ahmed M, Liu Z, Afzal KS, et al. Radiofrequency ablation: effect of surrounding tissue composition on coagulation necrosis in a canine tumor model. Radiology 2004; 230:761767.
24. Liszczak TM, Hedley-Whyte ET, Adams JF, et al. Limitations of tetrazolium salts in delineating infracted brain. Acta Neuropathol (Berl) 1984; 65:150-157.

25. Goldberg SN, Charboneau JW, Dodd GD III, et al. International Working Group on Image-Guided Tumor Ablation. Image-guided tumor ablation: proposal for standardization of terms and reporting criteria. Radiology 2003; 228:335-345.

26. Meloni F, Lazzaroni S, Livraghi T. Percutaneous ethanol injection: single session treatment. Eur J Ultrasound 2001; 13:107-115. 